\title{
Initial State Radiation Studies at BABAR
}

\author{
A. Petzold ${ }^{\mathrm{a}}$ \\ ${ }^{a}$ Institut für Kern- und Teilchenphysik, Technische Universität Dresden, \\ 01062 Dresden, Germany (on behalf of the BABAR Collaboration)
}

We present results from BABAR on events containing a hard radiated photon from the $e^{+} e^{-}$initial state and several exclusive final states. For the $\pi^{+} \pi^{-} \pi^{+} \pi^{-}$final state the cross section is measured for center-of-mass energies from 0.6 to $4.5 \mathrm{GeV}$. Resonant structures are studied and confirmed to be dominated by the $a_{1}(1260) \pi$, with a contribution from $f_{2}(1270) \rho(770)$. Similar studies are shown for $\pi^{+} \pi^{-} K^{+} K^{-}$and $K^{+} K^{-} K^{+} K^{-}$from their respective thresholds up to $4.5 \mathrm{GeV}$. From the $\pi^{+} \pi^{-} \pi^{0}$ final state the products of the branching fractions of the $\omega$ and $\phi$ mesons have been obtained and the cross section is measured from 1.05 to $3.00 \mathrm{GeV}$. In addition the $J / \psi$ branching fractions to all four final states have been measured.

\section{Introduction}

As theoretical predictions of $(g-2)$ of the muon and the running QED coupling constant depend on the precise knowledge of the production of hadronic final states at energies below $10 \mathrm{GeV}$, the interest in new measurements in this energy range has recently increased. Currently the predictions of the hadronic contributions to these two quantities are taken from direct $e^{+} e^{-}$measurements at low energies.

The use of initial state radiation (ISR) processes with hard photons at meson factories for hadron spectroscopy and the measurement of hadronic cross sections has been proposed in the past [1]. The advantage in using ISR events is that a scan of the entire effective center of mass energy range, the available energy after radiation of a hard photon, is performed in the same experiment. Thereby, uncertainties in the relative normalization when combining data from different experiments can be avoided.

The production cross section for a particular final state $f$ accompanied by a hard ISR photon can be written as

$\frac{d \sigma(s, x)}{d x}=W(s, x) \cdot \sigma_{f}(s(1-x))$.

It depends on the fraction $x=\frac{2 E_{\gamma}}{\sqrt{s}}$ of the beam energy $\sqrt{s}$ carried by the photon with energy $E_{\gamma}$ in the nominal center of mass frame and the $e^{+} e^{-}$ cross section $\sigma_{f}$. The radiator function $W(s, x)$ describes the energy spectrum of the virtual photons.

\section{The BABAR Detector}

The BABAR experiment operates at the PEP-II $e^{+} e^{-}$collider at a center of mass energy of $10.58 \mathrm{GeV}$. The BABAR detector is described in detail elsewhere [2]. A five-layer double-sided silicon vertex tracker and a 40-layer drift chamber located in a $1.5 \mathrm{~T}$ magnetic field, enable the measurement of parameter values of charged-particle tracks. Charged particle identity is established by means of an internally reflecting ring imaging Cherenkov detector (DIRC) and the specific energy loss $(d E / d x)$ measured in the tracking detectors. Photons are detected by an electromagnetic calorimeter consisting of $6580 \mathrm{CsI}(\mathrm{Tl})$ crystals. Muon identification and detection of long-lived neutral hadrons is provided by an instrumented flux return.

\section{The $\pi^{+} \pi^{-} \pi^{0}$ Final State}

The initial selection criteria of $e^{+} e^{-} \rightarrow$ $\pi^{+} \pi^{-} \pi^{0} \gamma$ candidate events require the detection of all final state particles inside the fiducial volume of the detector. Two tracks must originate from the interaction point, have transverse mo-

Contributed to QCD 05: 12th International QCD Conference: 20 Years of the QCD-Montpellier Conference, 07/04/2005--7/8/2005, Montpellier, France

Work supported in part by US Department of Energy contract DE-AC02-76SF00515 
menta above $100 \mathrm{MeV} / c$, must not be identified as kaons and be in a region of good detector acceptance. A candidate event has to contain at least three photons with energies above $100 \mathrm{MeV}$ and one of them is required to have a center of mass energy above $3 \mathrm{GeV}$. The ISR photon is assumed to be the one with the highest energy in the center of mass frame. Candidates for $\pi^{0} \mathrm{~s}$ are constructed from pairs of remaining photons. A kinematic fit is applied to the selected event, imposing energy and momentum conservation as well as constraining the invariant mass of the $\pi^{0}$ candidate. A detailed description of the analysis has been published in [3].

In order to extract the peak cross sections for $e^{+} e^{-}$annihilation into $\omega$ and $\phi$ mesons and the resonance parameters of excited $\omega$ states the background-subtracted $3 \pi$ invariant-mass spectrum was fit (Fig. 1). The fit includes a sum of contributions describing $\omega(782), \phi(1020)$, $\omega(1420)$, and $\omega(1650)$. The relative phases for $\omega(1420)$ and $\omega(1650)$ are fixed to 0 and $\pi$. The branching fraction products, masses, and widths obtained from the fit are:

$\mathcal{B}_{\omega e e} \mathcal{B}_{\omega 3 \pi}=(6.70 \pm 0.06 \pm 0.27) \times 10^{-5}$, $\mathcal{B}_{\phi e e} \mathcal{B}_{\phi 3 \pi}=(4.30 \pm 0.06 \pm 0.21) \times 10^{-5}$,

$\mathcal{B}_{\omega^{\prime} e e} \mathcal{B}_{\omega^{\prime} 3 \pi}=(0.82 \pm 0.05 \pm 0.06) \times 10^{-6}$,

$\mathcal{B}_{\omega^{\prime \prime} e e} \mathcal{B}_{\omega^{\prime \prime} 3 \pi}=(1.3 \pm 0.1 \pm 0.1) \times 10^{-6}$,

$M_{\omega^{\prime}}=(1350 \pm 20 \pm 20) \mathrm{MeV} / c^{2}$,

$M_{\omega^{\prime \prime}}=(1660 \pm 10 \pm 2) \mathrm{MeV} / c^{2}$,

$\Gamma_{\omega^{\prime}}=(450 \pm 70 \pm 70) \mathrm{MeV} / c^{2}$,

$\Gamma_{\omega^{\prime \prime}}=(230 \pm 30 \pm 20) \mathrm{MeV} / c^{2}$.

The fitted masses and widths of the $\omega^{\prime}$ and $\omega^{\prime \prime}$ mesons are model dependent but can be compared to the current estimates of these parameters by the PDG [4]: $M_{\omega^{\prime}}=(1400-1450) \mathrm{MeV} / c^{2}$, $M_{\omega^{\prime \prime}}=(1670 \pm 30) \mathrm{MeV} / c^{2}, \quad \Gamma_{\omega^{\prime}}=(180-$ 250) $\mathrm{MeV}, \Gamma_{\omega^{\prime \prime}}=(315 \pm 35) \mathrm{MeV}$. The present $B A B A R$ measurements are based on a significantly larger data sample for the reaction $e^{+} e^{-} \rightarrow$ $\omega^{\prime}, \omega^{\prime \prime} \rightarrow 3 \pi$ than the sample used for the PDG data.

The measured $e^{+} e^{-} \rightarrow \pi^{+} \pi^{-} \pi^{0}$ cross section in the mass region of 1.05 to $3.0 \mathrm{GeV} / c^{2}$ is in agreement with data from SND [5] for the mass region below $1.4 \mathrm{GeV} / c^{2}$, but in contradiction with measurements from DM2 [6].
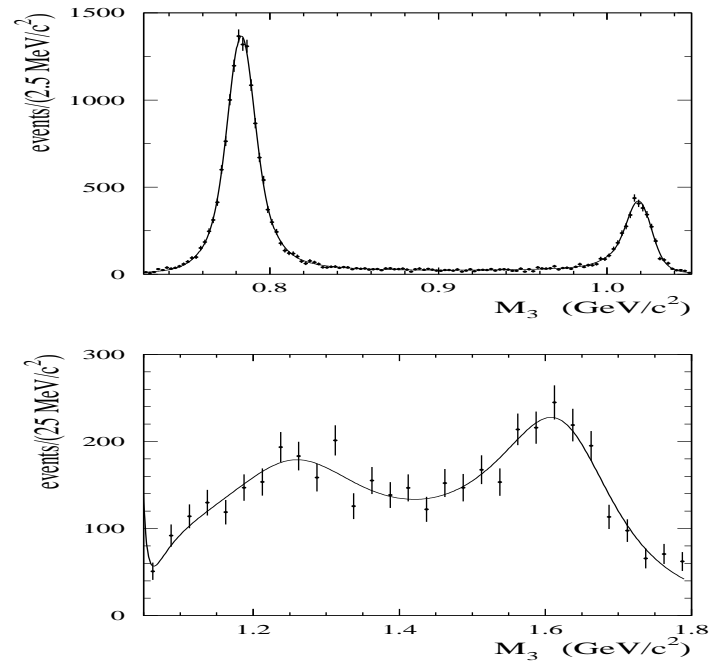

Figure 1. The background-subtracted $3 \pi$ mass spectrum and the result of fit for masses between 0.70 and $1.05 \mathrm{GeV} / c^{2}$ (top) and for masses from 1.05 to $1.80 \mathrm{GeV} / c^{2}$ (bottom).

\section{The $\pi^{+} \pi^{-} \pi^{+} \pi^{-}, \pi^{+} \pi^{-} K^{+} K^{-}$, and $\boldsymbol{K}^{+} \boldsymbol{K}^{-} \boldsymbol{K}^{+} \boldsymbol{K}^{-}$Final States}

At least four charged-particle tracks and one hard photon, assumed to be coming from the initial state, are required to select a candidate event. All tracks are subjected to a kaon identification procedure using energy loss in the tracking detectors and information from the DIRC. A kinematic fit is performed to assign an event to one of the three final state categories and to reject backgrounds. Details of the analysis procedure can be found in [7].

The cross section for $e^{+} e^{-} \rightarrow \pi^{+} \pi^{-} \pi^{+} \pi^{-}$is shown in Fig. 2 in comparison with all available $e^{+} e^{-}$data. Fig. 3 shows the cross section for the $\pi^{+} \pi^{-} K^{+} K^{-}$and $K^{+} K^{-} K^{+} K^{-}$final states. The $\pi^{+} \pi^{-} \pi^{+} \pi^{-}$and $\pi^{+} \pi^{-} K^{+} K^{-}$results agree with existing results, but are substantially more precise. The $4 K$ result is the first measurement of this quantity. The dominant systematic uncertainties include the luminosity measurement, tracking efficiencies, and acceptance losses. 


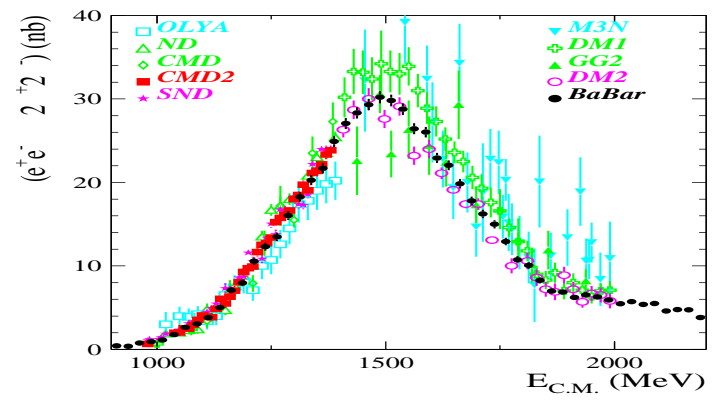

Figure 2. The $e^{+} e^{-} \rightarrow \pi^{+} \pi^{-} \pi^{+} \pi^{-}$cross section obtained by BABAR compared to all $e^{+} e^{-}$data.

\section{The $J / \psi$ Decays}

In all four invariant mass spectra the $J / \psi$ signal is clearly visible. Detailed plots of the $J / \psi$ signals for three and four pions are shown in Fig. 4.

In the case of three pions the following branching fraction product can be determined. $\Gamma(\mathrm{J} / \psi \rightarrow$ $\left.e^{+} e^{-}\right) \mathcal{B}\left(J / \psi \rightarrow \pi^{+} \pi^{-} \pi^{0}\right)=(0.1222 \pm 0.005 \pm$ $0.008) \mathrm{keV}$. Using a previous $B A B A R$ measurement of the electronic width of the $J / \psi[8]$, the $J / \psi \rightarrow 3 \pi$ branching fraction can be calculated to be $\mathcal{B}\left(J / \psi \rightarrow \pi^{+} \pi^{-} \pi^{0}\right)=(2.18 \pm 0.19) \%$. This is in substantial disagreement with the world average of $(1.47 \pm 0.13) \%$. However, it is in agreement with a recent BES result of $\mathcal{B}\left(J / \psi \rightarrow \pi^{+} \pi^{-} \pi^{0}\right)=$ $(2.10 \pm 0.12) \%$.

The bottom plot in Fig. 4 shows the $J / \psi$ signal in the $4 \pi$ final state. In addition a signal for the $\psi(2 S)$ is seen from the process $\psi(2 S) \rightarrow$ $J / \psi\left(\mu^{+} \mu^{-}\right) \pi^{+} \pi^{-}$where the muons are misidentified as pions. The branching fraction products are the following:

$\mathcal{B}\left(J / \psi \rightarrow \pi^{+} \pi^{-} \pi^{+} \pi^{-}\right) \Gamma\left(J / \psi \rightarrow e^{+} e^{-}\right)=$ $(1.95 \pm 0.14 \pm 0.13) \times 10^{-2} \mathrm{keV}$,

$\mathcal{B}\left(\psi(2 S) \rightarrow J / \psi \pi^{+} \pi^{-}\right) \mathcal{B}\left(J / \psi \rightarrow \mu^{+} \mu^{-}\right)$

$\Gamma\left(\psi(2 S) \rightarrow e^{+} e^{-}\right)=$

$(4.50 \pm 0.18 \pm 0.22) \times 10^{-2} \mathrm{keV}$.

Combined with the PDG values of $\Gamma(J / \psi \rightarrow$ $\left.e^{+} e^{-}\right), \Gamma\left(\psi(2 S) \rightarrow e^{+} e^{-}\right)$, and $\mathcal{B}\left(J / \psi \rightarrow \mu^{+} \mu^{-}\right)$ the branching fractions for $J / \psi$ and $\psi(2 S)$ can be derived:
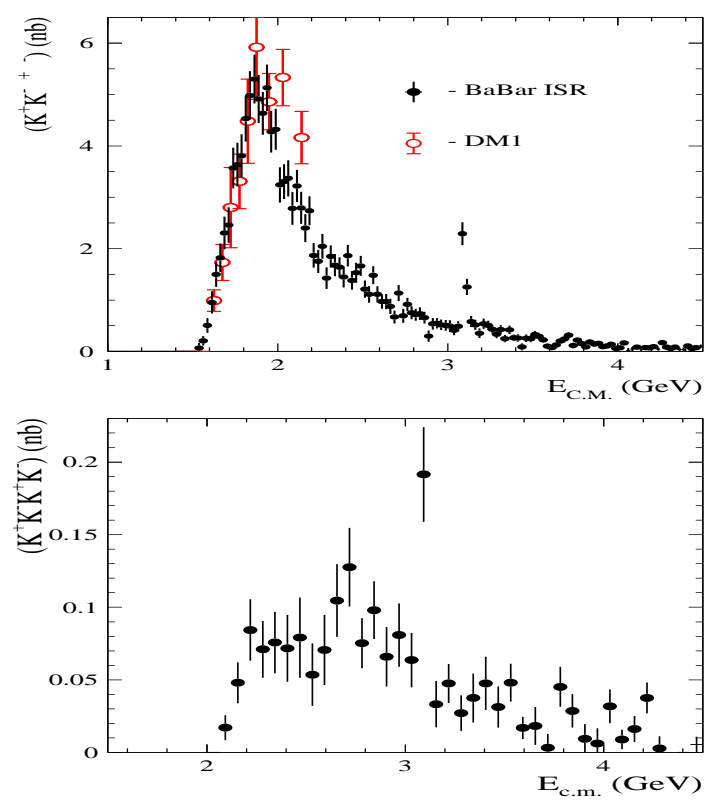

Figure 3. The $e^{+} e^{-} \rightarrow \pi^{+} \pi^{-} K^{+} K^{-}$(top) and $K^{+} K^{-} K^{+} K^{-}$(bottom) cross sections obtained from ISR at BABAR.

$\mathcal{B}\left(J / \psi \rightarrow \pi^{+} \pi^{-} \pi^{+} \pi^{-}\right)=$

$(3.70 \pm 0.27 \pm 0.36) \times 10^{-3}$,

$\mathcal{B}\left(\psi(2 S) \rightarrow J / \psi \pi^{+} \pi^{-}\right)=$

$0.361 \pm 0.015 \pm 0.037$.

For the final states containing kaons it is possible to measure the following branching fraction products:

$\mathcal{B}\left(J / \psi \rightarrow \pi^{+} \pi^{-} K^{+} K^{-}\right) \Gamma\left(J / \psi \rightarrow e^{+} e^{-}\right)=$ $(3.29 \pm 0.27 \pm 0.27) \times 10^{-2} \mathrm{keV}$,

$\mathcal{B}\left(J / \psi \rightarrow K^{+} K^{-} K^{+} K^{-}\right) \Gamma\left(J / \psi \rightarrow e^{+} e^{-}\right)=$ $(3.6 \pm 0.6 \pm 0.5) \times 10^{-3} \mathrm{keV}$.

Again combining these results with the world average for $\Gamma\left(J / \psi \rightarrow e^{+} e^{-}\right)$the respective $J / \psi$ branching fractions can be derived:

$\mathcal{B}\left(J / \psi \rightarrow \pi^{+} \pi^{-} K^{+} K^{-}\right)=$

$(6.25 \pm 0.50 \pm 0.62) \times 10^{-3}$,

$\mathcal{B}\left(J / \psi \rightarrow K^{+} K^{-} K^{+} K^{-}\right)=$

$(6.9 \pm 1.2 \pm 1.1) \times 10^{-4}$.

These branching fraction results agree with the PDG values, but are significantly more precise. 

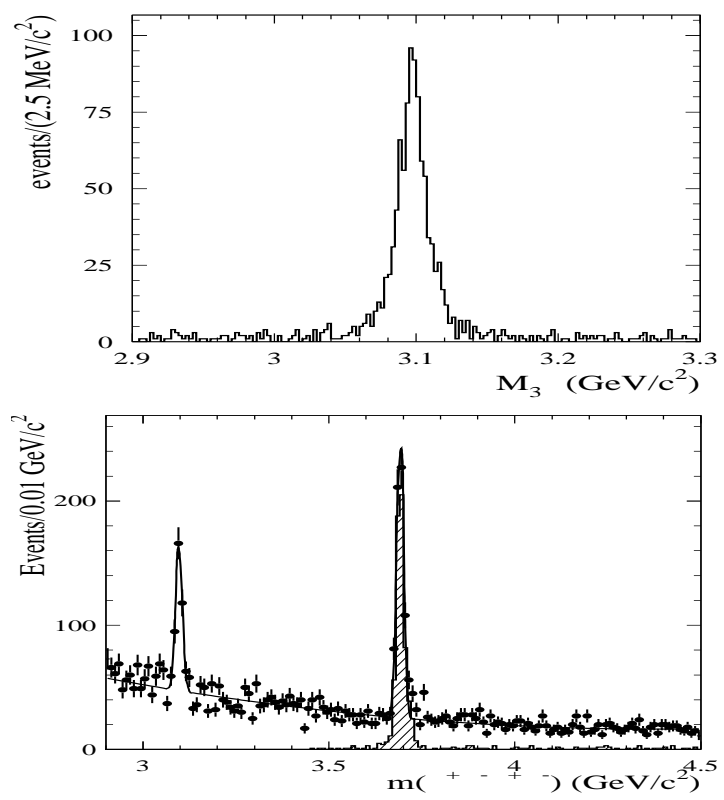

Figure 4. Signals from $J / \psi$ in $3 \pi$ (top) and $J / \psi$ and $\psi(2 S)$ in $4 \pi$ (bottom) final states.

\section{Conclusion}

Studies of hadronic cross sections using initial state radiation processes offer promising prospects at BABAR. The detector offers excellent detection efficiency and particle identification. Several exclusive channels have already been measured, and more are in progress. The presented $e^{+} e^{-} \rightarrow \pi^{+} \pi^{-} \pi^{0}, \pi^{+} \pi^{-} \pi^{+} \pi^{-}, K^{+} K^{-} \pi^{+} \pi^{-}$, $K^{+} K^{-} K^{+} K^{-}$cross sections cover the energy region from threshold to $4.5 \mathrm{GeV}$, which is a wider range than achieved by any other previous experiment. The systematic errors are similar to those of measurements of direct $e^{+} e^{-}$experiments. Radiative return to the $J / \psi$ and $\psi(2 S)$ resonances allows the precise determination of several branching fraction products.

\section{REFERENCES}

1. M. Benayoun et al., Mod. Phys. Lett. A14, 2605 (1999)

2. B. Aubert et al., BABAR Collaboration, Nucl. Instr. Methods Phys. Res., Sect. A 479, 1-116 (2002)

3. B. Aubert et al., BABAR Collaboration, Phys. Rev. D 70, 072004 (2004)

4. S. Eidelman et al., Review of Particle Physics, Phys. Lett. B 592, 1 (2004)

5. M.N. Achasov et al., SND Collaboration, Phys. Rev. D 66, 032001 (2002)

6. A. Antonielli et al., DM2 Collaboration, Z. Phys. C 56, 15 (1992)

7. B. Aubert et al., BABAR Collaboration, Phys. Rev. D 71, 052001 (2005)

8. B. Aubert et al., BABAR Collaboration, Phys. Rev. D 69, 011103 (2004) 